\title{
Electron Diffraction of Graphene-covered Protein Crystals at Room Temperature
}

Sercan Keskin ${ }^{1}$ and Niels de Jonge ${ }^{2}$

${ }^{1}$ INM - Leibniz Institute for New Materials, Saarbrücken, Germany, Saarbrücken, Germany, ${ }^{2}$ INM Leibniz Institute for New Materials, Saarbrücken, Germany

Electron crystallography has been used successfully to determine the three-dimensional structure of proteins using sub-micron thick crystals [1]. The state-of-the-art method to preserve the structure of protein crystals for transmission electron microscopy (TEM) is to freeze them rapidly in a thin layer of amorphous ice and conduct imaging/diffraction at cryogenic temperatures. Electron crystallography at cryogenic conditions has been particularly valuable to determine the structure of membrane proteins, where other methods such as X-ray crystallography are difficult to implement, with a resolution reaching beyond $2 \AA$ [2]. The main limitation of the method is electron beam-induced radiation damage during the data collection. High energy electrons disrupt the crystalline order; hence limit the attainable resolution [3]. Recently, it was shown that graphene encapsulation can maintain the structural integrity of microtubule proteins for TEM at room temperature. In the same study, nearly one order of magnitude beam damage mitigation was observed in the protofilament structure of microtubules by measuring the intensity decay at $0.2 \mathrm{~nm}^{-1}(5 \mathrm{~nm})$ spatial frequencies with graphene encapsulation compared to cryofixated microtubules in a hole on carbon foil [4].

In this study, we used graphene-coated TEM grids to cover the catalase crystals on amorphous carboncoated TEM grids, and acquired diffraction images to investigate the radiation damage at higher resolution structures of proteins than $5 \mathrm{~nm}$, which correspond to smaller atomic spacing and more prone to be damaged by the electron beam. Besides the beam damage mitigation effect of graphene on proteins, this method enables studying protein structure at biologically relevant temperatures. Figure 1 shows a schematic representation of the sample preparation for TEM (a), a typical TEM image (b), and a background-subtracted diffraction pattern (c) obtained from the crystals. The diffraction pattern of graphene obtained from the same sample region was also shown in Figure 1d to verify the presence of graphene covering the crystals.

Here, we discuss results regarding the structural stability of protein crystals with graphene-carbon encapsulation in TEM without resin-embedding, staining, or freezing. Observed Bragg peaks reaching $0.36 \mathrm{~nm}^{-1}(2.8 \mathrm{~nm})$ in the diffraction image (pointed by the blue arrow in Figure 1c) suggest that the crystallinity was preserved to some extent with graphene-carbon encapsulation. The intensity decay at these peaks as a function of cumulative electron dose was used to determine the critical dose before significant damage occurs. Figure 1e-h shows the diffraction images successively obtained from the same crystal region at increasing cumulative electron doses. As seen, the outermost peaks faded first. The peaks observed at a resolution of $0.2 \mathrm{~nm}^{-1}$ and higher completely disappeared beyond a cumulative dose of 98 $\mathrm{e}^{-} \AA^{-2}$, while the peaks observed at a lower resolution than $0.2 \mathrm{~nm}^{-1}$ did not exhibit a significant intensity change. 

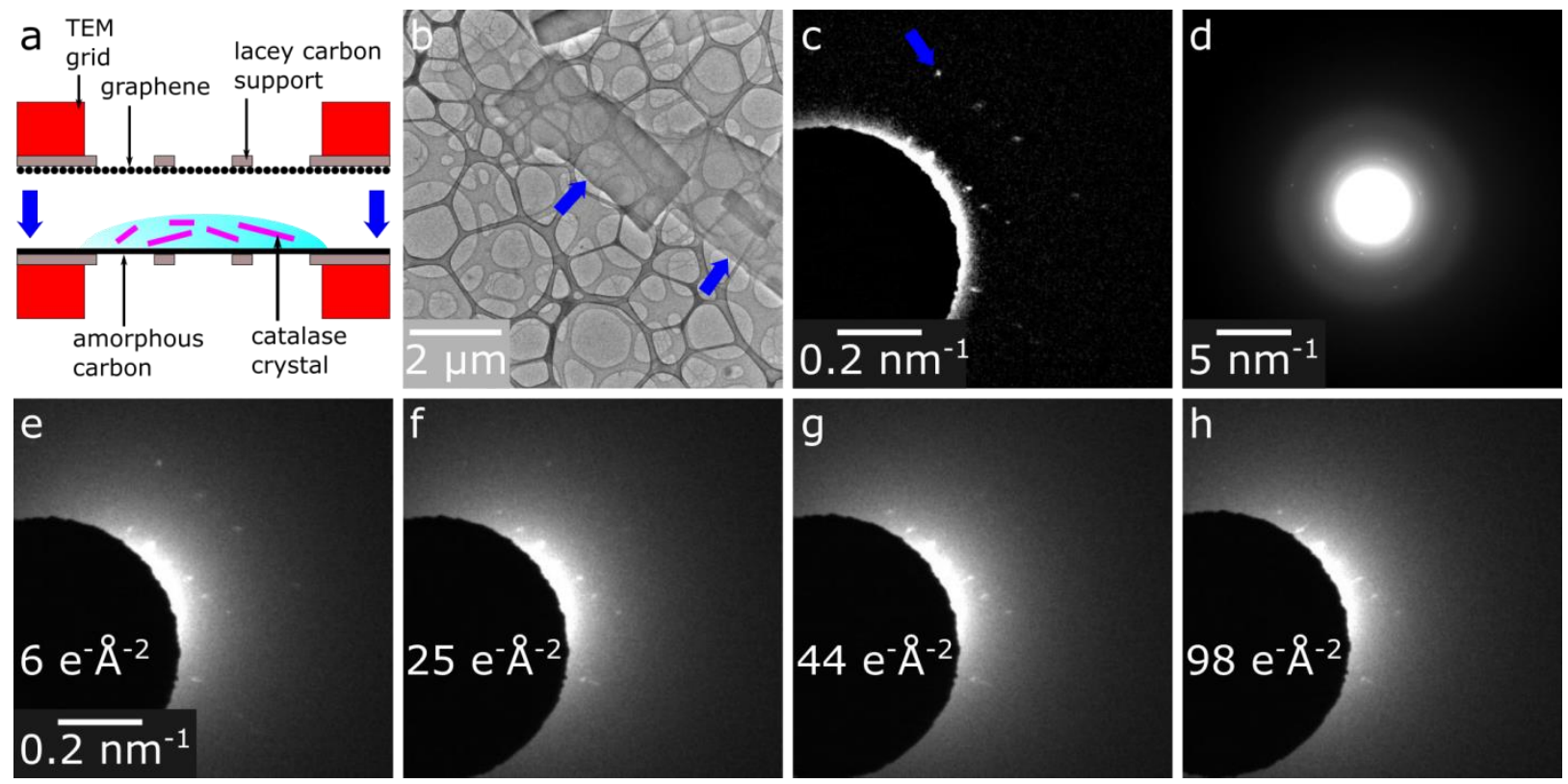

Figure 1. Electron diffraction of graphene-covered catalase crystals at room temperature. a) Schematic representation of the encapsulation. b) Transmission electron microscopy (TEM) image of the catalase crystals pointed by arrows. c) A typical electron diffraction image obtained from a catalase crystal using a beam size of $0.3 \mu \mathrm{m}$ at $200 \mathrm{keV}$. An electron flux of $1.2 \mathrm{e}-\AA-2 \mathrm{~s}-1$ was used with an exposure time of 5 $\mathrm{s}$ resulting in a total dose of $6 \mathrm{e}-\AA$ A- 2 . The diffraction image in (c) was subtracted by the same image after a Gaussian blur to increase the visibility of the Bragg peaks. d) Electron diffraction image obtained from the same crystal region as in (c) with a different camera length showing the peaks originating from graphene. e-h) Raw diffraction images (without background subtraction), successively obtained from the same crystal region at increasing cumulative electron doses. The cumulative electron dose is indicated on each image. The scale bar in (e) is $0.2 \mathrm{~nm}-1$ and the same for (e-h).

\section{References}

[1] R Henderson and P N T Unwin. Nature, 257 (1975) p. 28.

[2] T Gonen et al. Nature, 438 (2005) p. 633.

[3] R F Egerton. Micron, 119 (2019) p. 72.

[4] S Keskin and N de Jonge. Nano Lett, 18 (2018) p. 7435.

[5] We thank Eduard Arzt for his support through INM. 